\title{
Optical gain of two-dimensional hole gas by intersubband Raman transitions
}

\author{
Soichiro Tsujino ${ }^{\text {a) }}$ \\ Laboratory for Micro- and Nanotechnology, Paul Scherrer Institut, CH-5232 Villigen-PSI, Switzerland
}

(Received 18 February 2012; accepted 20 June 2012; published online 19 July 2012)

\begin{abstract}
Raman gain of a two-dimensional hole gas is theoretically studied. It is shown that a finite gain is expected for a $p$-type quantum well without introducing asymmetry via a Raman transition between confined hole states. The gain and loss in SiGe and Ge quantum wells on Si substrates by hole intersubband transitions are modeled using an experimentally validated 6-band $k \cdot p$ method combined with a design of a SiGe ridge waveguide on Si substrate. A net modal gain of $\sim 20 \mathrm{~cm}^{-1}$ with the pump intensity of $\sim 4 \mathrm{MW} \mathrm{cm}^{-2}$ is predicted for a device with multiple square Ge quantum well active layers by the proposed intersubband Raman transition. (C) 2012 American Institute of Physics. [http://dx.doi.org/10.1063/1.4737575]
\end{abstract}

\section{INTRODUCTION}

Optical components using Si-based materials have been intensely studied to surmount the potential bottleneck of the electrical interconnects in short and medium distances between microprocessor chips. ${ }^{1}$ Si-based photonic devices such as Si Raman laser, ${ }^{2}$ Ge laser, ${ }^{3}$ and modulator ${ }^{4}$ utilizing the interband transitions in Ge prepared on $\mathrm{Si}^{5,6}$ and defects ${ }^{7,8}$ or quantum-dot-embedded ${ }^{9} \mathrm{Si}$ light emitting diodes have been reported recently. A different approach utilizes optical transitions between confined states in high Ge-content SiGe and Ge quantum wells with large valence band energy offsets. In contrast to the interband transitions between conduction band and valence band, transition energies of the inter-subband transitions can be largely tailored by design. ${ }^{10-17}$ Electroluminescence from strain-compensated $\mathrm{Si}-\mathrm{SiGe}$ quantum cascade structure was reported, but lasing has remained to be a challenge because of the insufficient optical gain with respect to the waveguide loss, in particular from current injector layers. $^{14}$

In this work, optical gain of a two-dimensional hole gas in strain-compensated SiGe quantum wells generated by optical pumping is explored. In contrast to the electrically pumped structures, the absence of current injectors and associated waveguide loss is likely to offer a critical advantage to achieve lasing. Moreover, optical pumping scheme allows one to study basic optical transitions in simpler device structures. Here, a Raman transition in a three-level system formed in high-Ge content $p$-type SiGe wells is considered. Unlike $n$-type quantum wells studied in the past, ${ }^{18-22}$ large Raman gain is predicted in a square quantum well without introducing asymmetry by combining a TM-polarized transition between light-hole ( $\mathrm{LH})$ states with a TE-polarized transition between $\mathrm{LH}$ and heavy-hole $(\mathrm{HH})$ state. In $\mathrm{SiGe}$ systems, because of the large $\mathrm{HH}$ effective mass and the large intersubband linewidth broadening by the interfaceroughness scattering, ${ }^{15}$ the proposed Raman gain in square wells is more robust and advantageous than in asymmetric wells utilizing solely TM-polarized HH-transitions.

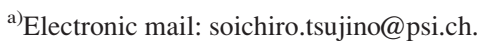

\section{INTERSUBBAND RAMAN GAIN IN p-TYPE SQUARE SiGe QUANTUM WELLS}

The intersubband Raman gain spectrum for a signal photon $(\mathrm{S})$ with energy $E_{\mathrm{S}}$ excited by a pump photon $(\mathrm{P})$ with energy $E_{\mathrm{p}}$ is given by the following equation when $E_{\mathrm{s}}$ is close to the transition energy $E_{32}$ between the third level (3) and the second level (2) and $E_{\mathrm{p}}$ is close to the transition energy $E_{13}$ between the ground level (1) and 3 at the same time,

$$
G_{R}\left(E_{s}, E_{p}\right)=\frac{4 \pi \alpha}{\eta_{s}} \omega_{s} z_{32}^{2} \operatorname{Re}\left[\frac{\left|e_{p}\right|^{2}}{\Gamma_{32} \Gamma_{13} \Gamma_{12}} \frac{\left(p_{1}-p_{3}\right)}{1+\left|e_{p}\right|^{2} /\left(\Gamma_{32} \Gamma_{12}\right)}\right] .
$$

In Eq. (1), $\alpha$ is the fine structure constant, $\eta_{\mathrm{s}}$ is the refractive index, $p_{\mathrm{n}}$ are hole concentrations in the $n$-th level, and $z_{32}$ is the dipole moment of the transition between 3 and 2 . The pump excitation is introduced as the Rabi frequency $e_{\mathrm{p}}$ given by $e_{0} r_{13} F_{\mathrm{p}} /(2 \hbar)$ with the elementary charge $e_{0}$, the dipole moment $r_{13}$ of the 1-to-3 transition, and the electric field $F_{\mathrm{p}}$ of the pump. In Eq. (1), following detunings were defined, $\quad \Gamma_{13}=\gamma_{13}-\mathrm{i} \Delta, \quad \Delta=\left(E_{31}-E_{\mathrm{p}}\right) / \hbar, \quad \Gamma_{23}=\gamma_{23}-\mathrm{i} \delta$, $\delta=\left(E_{32}-E_{\mathrm{s}}\right) / \hbar$, and $\Gamma_{12}=\gamma_{12}-\mathrm{i}(\Delta-\delta)$, with $\gamma_{\mathrm{nm}}$ the dephasing rate of the $n$-to- $m$ optical transition. Equation (1) is obtained from a steady state solution of the density-matrix within the rotating-wave approximation, solved together with the Maxwell's equation with the envelope-function approximation. ${ }^{18}$

In a $p$-type square quantum well, one can identify the first heavy hole level $\mathrm{H} 1$ as 1 , the first light hole level L1 as 2, and the second light hole level L2 as 3, see Figs. 1(a) and 2(a). In $n$-type quantum wells, spatial asymmetry is crucial to form a three-level system with finite $r_{13}$, but not in valence band. Whereas the $S$-transition with the matrix element $z_{32}$ is TM active (polarization perpendicular to the quantum well plane), the $P$-transition with the matrix element $r_{13}$ is TE active because of the interband nature of the HH to LH transition. The largest optical loss originates from the TM active absorption transition from $\mathrm{H} 1$ to the second heavy hole level $\mathrm{H} 2$, since the energy difference $\delta E$ between the H1-to- $\mathrm{H} 2$ transition energy $E_{\mathrm{H} 1-\mathrm{H} 2}$ and $E_{3}$ (the L2-to-L1 transition 


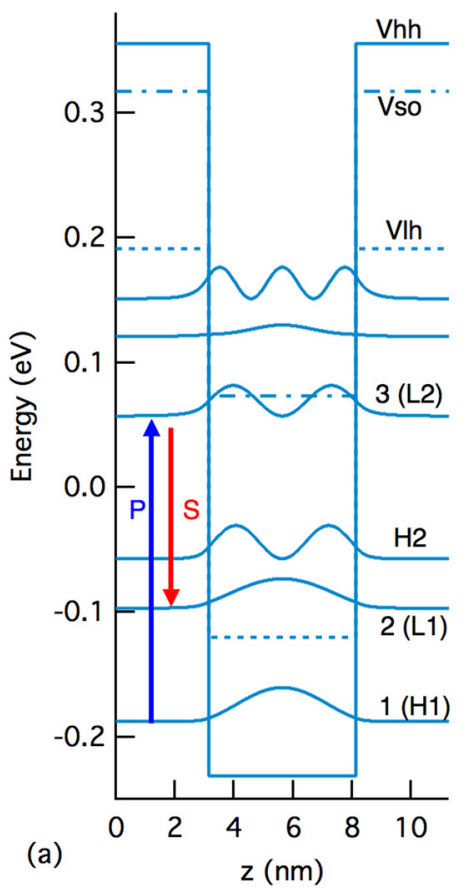

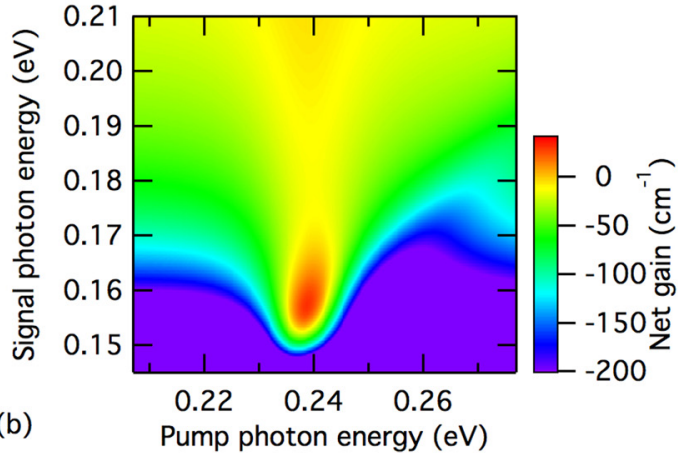

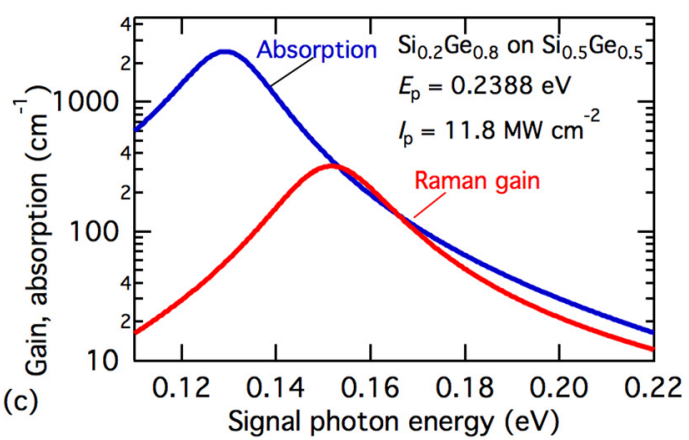

FIG. 1. (a) Hole energy levels of QW1 at $k=0$ for a $\mathrm{Si}_{0.2} \mathrm{Ge}_{0.8}$ quantum well $(5 \mathrm{~nm})$ separated by $3 \mathrm{~nm}$-thick Si barriers. $\mathrm{Si}_{0.5} \mathrm{Ge}_{0.5}$ substrate is assumed. (b) Net optical gain as a function of the pump photon energy and signal photon energy with the pump intensity of $11.9 \mathrm{MW}$ $\mathrm{cm}^{-2}$ calculated by 6-band $k \cdot p$ method. (c) Absorption and gain when pump photon energy is $0.2388 \mathrm{eV}$ with the same pump intensity assumed in (b). energy) is small. In the case of strain-compensated SiGe quantum wells, $\delta E$ is in the order of tens of $\mathrm{meV}$ for nearly all the range of Ge content. However, the non-zero $\delta E$ makes the net gain finite at sufficiently high pump excitation intensity. In the simulation shown below, it is shown that the required pump excitation intensity to reach transparency and lasing is moderate.

The quantum well in Figure 1(a), QW1, is a square $\mathrm{Si}_{0.2} \mathrm{Ge}_{0.8}$ well with $\mathrm{Si}$ barriers strain-compensated to a $\mathrm{Si}_{0.5} \mathrm{Ge}_{0.5}$ pseudo substrate, a material combination of which growth and optical transitions were studied in Refs. 13-15, 21, and 22. Fig. 1(a) depicts the energy levels at the $\Gamma$-point where the in-plane wave vector $k=0$, calculated by a 6-band $\boldsymbol{k} \cdot \boldsymbol{p}$ method. The band-offsets, deformation potentials, and Luttinger parameters used in the calculation were validated previously by comparisons with experiments such as the valence band intersubband absorption spectra including the LH-HH mixing, ${ }^{16,17}$ high-peak-to-value ratio hole resonant tunneling in $\mathrm{SiGe} / \mathrm{Ge}$ double-barrier diode, ${ }^{23}$ and directgap interband optical transition spectra in Ge quantum wells. ${ }^{6}$ Hole concentration per quantum well equal to $0.5 \times 10^{12} \mathrm{~cm}^{-2}$ was assumed. The gain and loss are obtained by integrating those for each $k$-state within $|k|$ below the Fermi wave vector $k_{\mathrm{F}}$.

To estimate the dephasing rates $\gamma_{\mathrm{nm}}$, experimental absorption spectra of $\mathrm{Si}_{0.2} \mathrm{Ge}_{0.8}$ quantum wells on $\mathrm{Si}_{0.5} \mathrm{Ge}_{0.5}$

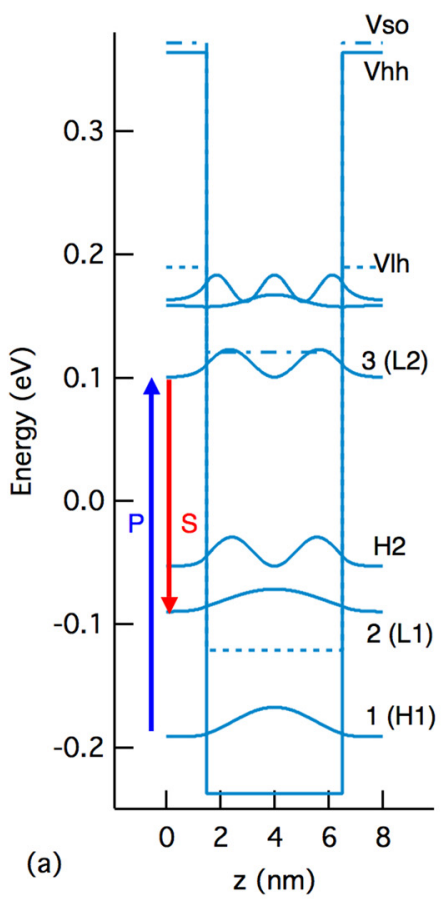

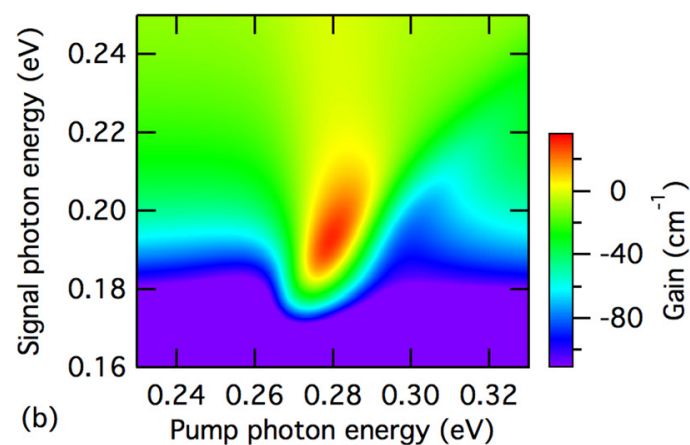

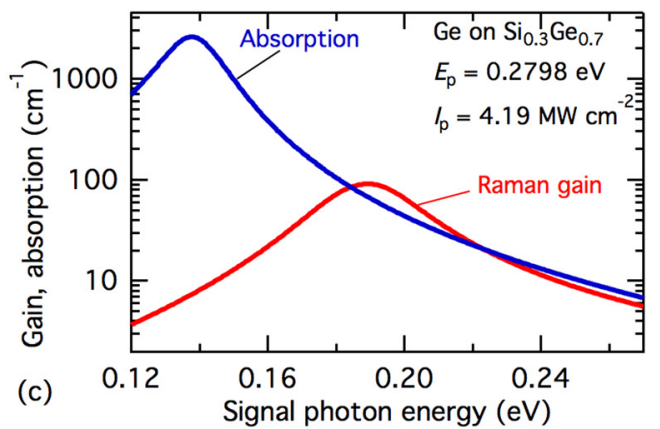

FIG. 2. (a) Hole energy levels of QW2 at $k=0$ for a Ge quantum well $(5 \mathrm{~nm})$ separated by $\mathrm{Si}_{0.8} \mathrm{Ge}_{0.2}$ barriers $(3 \mathrm{~nm}) . \mathrm{Si}_{0.3} \mathrm{Ge}_{0.7}$ substrate is assumed. (b) Net optical gain as a function of the pump photon energy and signal photon energy with the pump intensity of $4.19 \mathrm{MW}$ $\mathrm{cm}^{-2}$ calculated by 6-band $k \cdot p$ method. (c) Absorption and gain coefficient when pump photon energy is $0.2798 \mathrm{eV}$ with the same pump intensity assumed in (b). 
pseudo-substrates in Ref. 17 with the hole concentration of $1 \times 10^{12} \mathrm{~cm}^{-2}$ were consulted. The observed linewidth broadening (half width at the half maximum) of the H1-H2 transitions in $4.5 \mathrm{~nm}$-thick and $5.5 \mathrm{~nm}$-thick wells were $21 \mathrm{meV}$ and $12 \mathrm{meV}$, respectively. The linewidth broadening of the H1-L2 transitions was $16 \mathrm{meV}$ for $4.5 \mathrm{~nm}$-thick well, and $11 \mathrm{meV}$ for $5.5 \mathrm{~nm}$ well, respectively, whereas the linewidth of H1-L1 transitions was approximately same as H1H2. Comparing these values with the linewidth of calculated absorption spectra for the same hole concentration, it was found that the $k$-dispersion of the transition energy increased linewidth of the H1-H2 transition by (1-2) meV for both wells, and the linewidth of the H1-L2 transition by $6 \mathrm{meV}$ and $11 \mathrm{meV}$ for $5.5 \mathrm{~nm}$ - and $4.5 \mathrm{~nm}$-thick wells, respectively. Therefore, $\gamma_{\mathrm{H} 1-\mathrm{H} 2}, \gamma_{\mathrm{H} 1-\mathrm{L} 2}$, and $\gamma_{\mathrm{H} 1-\mathrm{L} 1}$ were assumed to be $10 \mathrm{meV}$. There were no direct absorption data for the L1-L2 transition, so $\gamma_{\mathrm{L} 1-\mathrm{L} 2}$ was approximated by $\left(\gamma_{\mathrm{H} 1-\mathrm{L} 1}+\gamma_{\mathrm{H} 1-\mathrm{L} 2}\right) / 2$.

Transient population in excited levels under optical pumping can modify the gain and loss. It can also cause the re-absorption of the $S$-photon. However, unlike III-V semiconductor case, it is not important in SiGe wells for excitation intensity of $2-20 \mathrm{MW} \mathrm{cm}^{-2}$ considered in the following. Therefore this effect was neglected. In fact, the optical excitation rate, which is below $2\left|e_{\mathrm{p}}\right|^{2} / \gamma_{31}$ and at most $0.4 \mathrm{ps}^{-1}$ for $20 \mathrm{MW} \mathrm{cm}{ }^{-2}$ with $\gamma_{31}$ of $10 \mathrm{meV}$ and $r_{13}$ of $0.39 \mathrm{~nm}$, is an order of magnitude smaller than expected L2level relaxation lifetime of $7-10 \mathrm{ps}^{-1}$ (Refs. 24 and 25).

\section{GAIN AND LOSS IN STRAIN-COMPENSATED SiGe-Si AND Ge-SiGe WELLS}

Figure 1(b) shows the spectrum of the net gain, $G_{\mathrm{R}}$ subtracted by the H1-H2 absorption, calculated for QW1 for TE-polarized pump with $E_{\mathrm{p}}$ near $0.24 \mathrm{eV}$ and TM-polarized signal with $E_{\mathrm{s}}$ around $0.16 \mathrm{eV}$, when the well is excited with the pump intensity $I_{\mathrm{p}}$ of $11.9 \mathrm{MW} \mathrm{cm} \mathrm{cm}^{-2}$. Fig. 1(c) shows $G_{\mathrm{R}}$ and the $\mathrm{H} 1-\mathrm{H} 2$ absorption separately as a function of $E_{\mathrm{s}}$ at the same $I_{\mathrm{p}}$ with $E_{\mathrm{p}}$ of $0.239 \mathrm{eV}$. A finite net gain of $30 \mathrm{~cm}^{-1}$ is obtained at $E_{\mathrm{s}}$ of $0.1573 \mathrm{eV}$ with this excitation condition, despite the large absorption by the $\mathrm{H} 1-\mathrm{H} 2$ transition centered at $\sim 0.13 \mathrm{eV}$, because of the finite detuning of the L2-L1 transition from the $\mathrm{H} 1-\mathrm{H} 2$. This $E_{\mathrm{s}}$ is more than $10 \mathrm{meV}$ larger than $E_{\mathrm{L} 2-\mathrm{L} 1}$, since the loss by the H1-H2 transition absorption decreases rapidly at higher energy.

Increasing the energy separation $\delta E$ between $E_{\mathrm{H} 1-\mathrm{H} 2}$ and $E_{\mathrm{L} 2-\mathrm{L} 1}$ without degrading the Raman transition matrix elements is a key to lower the pump intensity. While designs including coupled quantum wells or asymmetric wells are common in III-V structures, their effectiveness is limited for $\mathrm{SiGe}$ structures due to the interface-roughness-induced linewidth broadening of the transitions. ${ }^{15}$ As an alternative strategy, square wells with different Ge contents were considered, which lead to the finding that QW2 shown in Fig. 2(a), a $5 \mathrm{~nm}$-thick square Ge well with $\mathrm{Si}_{0.8} \mathrm{Ge}_{0.2}$ barriers on a $\mathrm{Si}_{0.3} \mathrm{Ge}_{0.7}$ substrate, exhibits $\delta E$ of $\sim 50 \mathrm{meV}$. Both of the matrix elements, $r_{31}$ and $z_{32}$, also increased in the Ge well, see Table I. These are a consequence of the larger Luttinger parameters in $\mathrm{Ge}$ than in $\mathrm{Si}$. Although less studied than low Ge-content structures, ${ }^{13-17}$ high quality strain com-
TABLE I. Parameters of the strain-compensated quantum wells QW1 and QW2. $I_{\text {pump }}$ is the intensity required to achieve the net gain of $30 \mathrm{~cm}^{-1}$ at the signal photon energy of $E_{\mathrm{s}}$, when the pump photon energy was $E_{\mathrm{p}}$. Transition energies at $k=0$ and dipole moments (square root of the squared average over $k_{\mathrm{F}}$ ) are also given.

\begin{tabular}{lcc}
\hline \hline Sample & $\mathrm{QW} 1$ & $\mathrm{QW} 2$ \\
\hline Layers & $\mathrm{Si} \mathrm{Si}_{0.2} \mathrm{Ge}_{0.8}$ & $\mathrm{Si}_{0.8} \mathrm{Ge}_{0.2} / \mathrm{Ge}$ \\
thickness $(\mathrm{nm})$ & $3 / 5$ & $3 / 5$ \\
Substrate & $\mathrm{Si}_{0.5} \mathrm{Ge}_{0.5}$ & $\mathrm{Si}_{0.3} \mathrm{Ge}_{0.7}$ \\
$E_{31}, E_{\mathrm{L} 2-\mathrm{H} 1}(\mathrm{eV})$ & 0.245 & 0.292 \\
$E_{32}, E_{\mathrm{L} 2-\mathrm{L} 1}(\mathrm{eV})$ & 0.154 & 0.190 \\
$E_{\mathrm{H} 1-\mathrm{H} 2}(\mathrm{eV})$ & 0.130 & 0.138 \\
$r_{31}(\mathrm{~nm})$ & 0.41 & 0.42 \\
$z_{32}(\mathrm{~nm})$ & 2.16 & 2.63 \\
$\sqrt{r_{31} z_{32}(\mathrm{~nm})}$ & 1.02 & 1.17 \\
$z_{\mathrm{H} 1-\mathrm{H} 2}(\mathrm{~nm})$ & 1.11 & 1.14 \\
$E_{\mathrm{P}}(\mathrm{eV})$ & 0.2388 & 0.2798 \\
$E_{\mathrm{S}}(\mathrm{eV})$ & 0.1573 & 0.1928 \\
$I_{\mathrm{pump}}\left(\mathrm{MW} \mathrm{cm}{ }^{-2}\right)$ & 11.9 & 4.19 \\
\hline \hline
\end{tabular}

pensated Ge-SiGe structures with high average Ge content can be prepared, for example, by low-energy plasmaenhanced chemical vapor deposition method. ${ }^{6,28}$ As a result of the enhanced $\delta E$, the required $I_{\mathrm{p}}$ is $4.19 \mathrm{MW} \mathrm{cm}{ }^{-2}$ to obtain the net gain of $30 \mathrm{~cm}^{-1}$ for $E_{\mathrm{p}}$ of $0.278 \mathrm{eV}$ and $E_{\mathrm{s}}$ of $0.193 \mathrm{eV}$, Figs. 2(b) and 2(c). The $I_{\mathrm{p}}$ here is larger than the threshold excitation intensity value observed for an $n$-type InGaAs midinfrared Raman laser ${ }^{21,25}$ because of the smaller matrix elements in SiGe, especially that of the pump transition $r_{13}$. Still, the required $I_{\mathrm{p}}$ and the intensity absorbed in the quantum well (below $\sim 100 \mathrm{~kW} \mathrm{~cm}^{-2}$ ) are well below the values which strain-compensated $\mathrm{SiGe}$ structure cannot widthstand. ${ }^{25}$ The calculated pump intensity required to obtain the net gain of $\sim 300 \mathrm{~cm}^{-1}$ was found to be $17 \mathrm{MW}$ $\mathrm{cm}^{-2}$ (with the differential gain of $21.5 \mathrm{~cm} / \mathrm{MW}$ ) with only a factor of $\sim 4$ higher intensity than that required for $\sim 30 \mathrm{~cm}^{-1}$. This is because the $\mathrm{H} 1-\mathrm{H} 2$ absorption remains unchanged, whereas the Raman gain increases steeply with the pump intensity. Equation (1) shows that the Raman gain is proportional to the hole concentration $p$. However, simulations for $p$ larger than $\sim 3 \times 10^{11} \mathrm{~cm}^{-2}$ showed that the pump intensity required to obtain the net gain of $30 \mathrm{~cm}^{-1}$ decreases sub-linearly with the increase of $p$ and saturates to $\sim 3 \mathrm{MW} \mathrm{cm}^{-2}$ above $9 \times 10^{11} \mathrm{~cm}^{-2}$. This is due to the simultaneous increase of the H1-H2 absorption-loss and the shift of $E_{32}\left(E_{\mathrm{L} 2-\mathrm{L} 1}\right)$ to lower energy (hence larger absorption) at larger $k$.

\section{SiGe WAVEGUIDE DESIGN ON GRADED BUFFER SUBSTRATES}

As reported in Ref. 14, one can design a SiGe waveguide structure on a SiGe relaxed buffer substrate prepared on a Si substrate. Fig. 3 shows one example using 250 period of QW2 as the active layer. The quantum wells are prepared on a $3.5 \mu \mathrm{m}$-thick stepped graded buffer on a Si substrate (terminated with a $0.5 \mu$ m-thick $\mathrm{Si}_{0.3} \mathrm{Ge}_{0.7}$ layer) and cladded by a $0.5 \mu \mathrm{m}$-thick $\mathrm{Si}_{0.3} \mathrm{Ge}_{0.7}$ layer. The individual barriers are tensile strained and the wells are compressively strained 


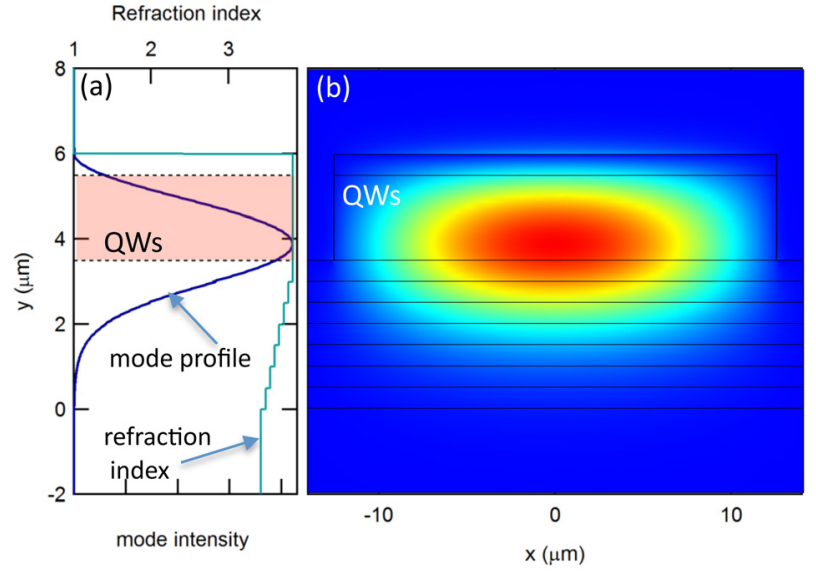

FIG. 3. (a) Mode profile of a fundamental TM mode at the photon energy of $0.195 \mathrm{eV}$ in a SiGe waveguide on a Si substrate with $2 \mu$ m-thick multiple Ge quantum wells (QW2) as the active layer (marked by light red box). The layers between $\mathrm{y}=0$ and $3.5 \mu \mathrm{m}$ are step-graded relaxed buffer terminated by a $0.5 \mu$ m-thick $\mathrm{Si}_{0.3} \mathrm{Ge}_{0.7}$ layer. The active layer is capped by a $0.5 \mu \mathrm{m}$ thick $\mathrm{Si}_{0.3} \mathrm{Ge}_{0.7}$ layer. (b) Cross-sectional mode profile in a $28 \mu$ m-wide waveguide ridge of the TM mode depicted in (a).

because of the large lattice mismatch between $\mathrm{Si}$ and Ge. Still, one can grow such SiGe structures when the layers are strain-compensated with respect to the relax buffer substrate; for example, when the average Ge content of the unit structure (a barrier and a well) is the same as the Ge content of the relaxed SiGe layer underneath the multi-layers, as long as the thickness of the individual layers are below the critical relaxation thickness. Successful growth of such high Ge-content strain-compensated superlattices and quantum cascade structures with excellent qualities by using the low-temperature molecular beam epitaxy $y^{15,26,27}$ and the low-energy plasma-enhanced chemical vapor desposition ${ }^{28}$ have been already demonstrated.

The guided mode in Figure 3 was calculated by using a finite element electromagnetic wave simulator (COMSOL Multiphysics) at the photon energy of $0.195 \mathrm{eV}$ for a $28 \mu \mathrm{m}$-wide ridge waveguide. The $62 \%$ overlap of the fundamental TM mode with the multiple QW2 active layers with $2 \mu \mathrm{m}$ total thickness was obtained for this design. This modal overlap can be further increased by using a Ge-cladding. ${ }^{14}$ End mirrors can be fabricated by reactive-ion etching ${ }^{27}$ to form a Fabry-Perot cavity. Assuming a waveguide length of $\sim 2 \mathrm{~mm}$ or above (with the facet reflectivity of $\sim 0.3$ ), the reflection loss at the mirror facets is estimated to be below $\sim 6 \mathrm{~cm}^{-1}$. Since the free carrier absorption by the residual impurities with the concentration in the order of $10^{16} \mathrm{~cm}^{-3}$ and the absorption by phonons in the considered wavelength range are both small (below $\sim 1 \mathrm{~cm}^{-1}$ ), ${ }^{25,29-31}$ the loss may originate from the scattering in the relaxed buffer substrate. Clarification and experimental investigation of the midinfrared waveguide loss of relaxed buffer substrates, especially those with high Ge content, require future study. Nevertheless, these consideration suggests that one can achieve transparency in the ridge waveguide SiGe Raman laser structures at the $I_{\mathrm{p}}$ values assumed for QW1 and 2 in Figs. 1 and 2, when the net gain of the active layer is in the order of $20-30 \mathrm{~cm}^{-1}$.

\section{CONCLUSION}

Motivated by the quest for the integration of optics into $\mathrm{Si}$ electronics, considerable effort has been expended in the past to the development of an electrically pumped Si laser. As a step toward that goal, the present work proposed to study an optically pumped device and provided a design of a test device, which allows for an experimental investigation of the gain and loss with a working Si-laser, albeit optically pumped operation. In particular, optically pumped Si-laser structures consisting of square $\mathrm{SiGe}$ and Ge quantum well active layers and SiGe waveguides were considered and analyzed theoretically. With the state-of-the-art crystal growth technology for the thick strain-compensated SiGe multi-layers, it is feasible to fabricate such devices. Predicted required pump intensities to achieve transparency and lasing were moderate that can be obtained by using a standard pulsed laser system in combination of parametric conversion. Being based on square quantum wells, the linewidth broadening of the proposed pump and gain transitions by the interface-roughness scattering is minimal. Therefore, the predicted gain is likely to be more robust than previously proposed gain structures based on the transitions in the superlattice states and quantum cascade states either in valence band or in conduction band.

\section{ACKNOWLEDGMENTS}

The author acknowledges S. Fukatsu for stimulating discussions on the optical gain in Si-based structures, J. Faist for helpful discussions on the Raman gain on symmetric and asymmetric SiGe structures, and M. Scheinert and H. Sigg for discussion on asymmetric SiGe structures on low-Ge substrates. This work was partially supported by the Swiss National Foundation.

${ }^{1}$ D. A. B. Miller, Proc. IEEE 97, 1166 (2009).

${ }^{2}$ H. Rong, S. Xu, Y.-H. Kuo, V. Sih, O. Cohen, O. Raday, and M. Paniccia, Nat. Photonics 1, 232 (2007).

${ }^{3}$ J. Liu, X. Sun, R. Camacho-Aguilera, L. C. Kimerling, and J. Michel, Opt. Lett. 35, 679 (2010)

${ }^{4}$ J. E. Roth, O. Fidaner, R. K. Schaevitz, Y.-H. Kuo, T. I. Kamins, J. S. Harrier, and D. A. B. Miller, Opt. Express 15, 5851 (2007).

${ }^{5}$ Y.-H. Kuo, Y.-K. Lee, Y. Ge, S. Ren, J. E. Roth, T. I. Kamins, D. A. B. Miller, and J. S. Harries, Nature (London) 437, 1334 (2005).

${ }^{6}$ S. Tsujino, H. Sigg, G. Mussler, D. Chrastina, and H. von Känel, Appl. Phys. Lett. 89, 262119 (2006).

${ }^{7}$ S. G. Cloutier, P. A. Kossyrev, and J. Xu, Nature Mater. 4, 887 (2005).

${ }^{8}$ J. Bao, M. Tabbal, T. Kim, S. Charnvanichborikarn, J. S. Williams, M. J. Aziz, and F. Cappasso, Opt. Express 15, 6727 (2007).

${ }^{9}$ Y. Yasutake, J. Igarashi, N. Tana-ami, and S. Fukatsu, Opt. Express 19, 16739 (2009).

${ }^{10}$ G. Dehlinger, L. Diehl, U. Gennser, H. Sigg, J. Faist, K. Ensslin, D. Grützmacher and E. Muller, Science 290, 2277 (2000).

${ }^{11}$ I. Bormann, K. Brunner, S. Hackenbuchner, G. Abstreiter, S. Schmult, and W. Wegscheider, Appl. Phys. Lett. 83(26), 5371 (2003).

${ }^{12}$ S. A. Lynch, R. Bates, D. J. Paul, D. J. Norris, A. G. Cullis, Z. Ikonic, R. W. Kelsall, P. Harrison, D. D. Arnone, and C. R. Pidgeon, Appl. Phys. Lett. 81, 1543 (2002).

${ }^{13}$ L. Diehl, S. Mentese, E. Müller, D. Grützmacher, H. Sigg, U. Gennser, I. Sagnes, Y. Campidelli, O. Kermarrec, D. Bensahel, and J. Faist, Appl. Phys. Lett. 81, 4700 (2002).

${ }^{14}$ S. Tsujino, H. Sigg, M. Scheinert, D. Grutzmacher, and J. Faist, IEEE J. Sel. Top. Quantum Electron. 12, 1642 (2006).

${ }^{15}$ S. Tsujino, A. Borak, E. Müller, M. Scheinert, C. V. Falub, H. Sigg, D. Grützmacher, M. Giovannini, and J. Faist, Appl. Phys. Lett. 86, 062113 (2005). 
${ }^{16}$ T. Fromherz, M. Meduna, G. Bauer, A. Borak, C. V. Falub, S. Tsujino, H. Sigg, and D. Grützmacher, J. Appl. Phys. 98, 044501 (2005).

${ }^{17} \mathrm{~S}$. Tsujino, A. Borak, C. Falub, T. Fromherz, L. Diehl, H. Sigg, and D. Grutzmacher, Phys. Rev. B 72, 153315 (2005).

${ }^{18}$ M. Troccoli, A. Belyanin, F. Cappasso, E. Cubukcu, D. L. Sivco, and A. Y. Cho, Nature (London) 433, 845 (2005).

${ }^{19}$ O. Gauthier-Lafaye, P. Boucaud, F. H. Julien, S. Sauvage, J. M. Lourtioz, V. Thierry-Mieg, and R. Planel, Appl. Phys. Lett. 71, 3619 (1997).

${ }^{20}$ H. C. Liu, C. Y. Song, Z. R. Wasilewski, A. J. SpringThorpe, J. C. Cao, C. Dharma-wardana, G. C. Aers, D. J. Lockwood, and J. A. Gupta, Phys. Rev. Lett. 90, 077402 (2003).

${ }^{21}$ M. Scheinert, H. Sigg, S. Tsujino, M. Giovannini, and J. Faist, Appl. Phys. Lett. 91, 131108 (2007).

${ }^{22}$ J. B. Khurgin, and H. C. Liu, Phys. Rev. B 74, 035317 (2006).

${ }^{23}$ S. Tsujino, N. Usami, A. Weber, G. Mussler, V. Shushunova, D. Grützmacher, Y. Azuma, and K. Nakajima, Appl. Phys. Lett. 91, 032104 (2007).

${ }^{24}$ K. Reimann, R. A. Kaindl, and M. Woerner, Phys. Rev. B 65, 045302 (2001).
${ }^{25}$ M. Scheinert, Ph.D. dissertation, University of Neuchatel, 2007.

${ }^{26}$ S. Tsujino, S. Mentese, L. Diehl, E. Müller, B. Haas, D. Bächle, S. Stutz, D. Grützmacher, Y. Campidelli, O. Kermarrec, and D. Bensahel, Appl. Surf. Sci. 224, 337 (2004).

${ }^{27}$ A. Borak, S. Tsujino, H. Sigg, C. V. Falub, M. Scheinert, L. Diehl, D. Grutzmacher, E. Muller, U. Gennser, Y. Campidelli, O. Kermarrec, D. Bensahel, and J. Faist, Mater. Res. Soc. Symp. Proc. 832, F4.2.1 (2005).

${ }^{28}$ M. Bonfanti, E. Grilli, M. Guzzi, M. Virgilio, G. Grosso, D. Chrastina, G. Isella, and H. von Känel, Phys. Rev. B 78, 041407 (2008).

${ }^{29}$ Landolt-Börnstein: Numerical Data and Functional Relationships in Science and Technology - New Series, Group III, Semiconductors, Subvolume a Vol. 17, edited by O. Madelung (Spinger-Verlag, Berlin 1982), p. 389 and 404.

${ }^{30}$ D. K. Schroder, R. N. Thomas, and J. C. Swartz, IEEE Trans. Electron Devices ED-25, 254 (1978)

${ }^{31}$ The dominant "free carrier absorption" by the carriers in the active layer is the $\mathrm{H} 1-\mathrm{H} 2$ intersubband absorption that was already accounted for in Figs. 1 and 2 . 